EFFECTS OF FRAILTY AND COGNITIVE IMPAIRMENT ON ADL DISABILITY: IMPLICATIONS ON COMPRESSION OF MORBIDITY

Q. Xue ${ }^{1}$, J. Tian, $\mathrm{MS}^{2}$, M.C. Carlson, $\mathrm{PhD}^{3}$, K. Bandeen-Roche, $\mathrm{PhD}^{4}$, 1. Johns Hopkins University School of Medicine, Balitimore, Maryland, United States, 2. Department of Biostatistics, Bloomberg School of Public Health, Johns Hopkins University, Baltimore, MD, USA; Center on Aging and Health, Johns Hopkins Medical Institutions, Baltimore, MD, USA, 3. Department of Epidemiology, Bloomberg School of Public Health, Johns Hopkins University, Baltimore, MD, USA, 4. Department of Biostatistics, Bloomberg School of Public Health, Johns Hopkins University, Baltimore, MD, USA; Center on Aging and Health, Johns Hopkins Medical Institutions, Baltimore, MD, USA

Using data from 5209 NHATS subjects living in the community or residential care setting, we assessed the associations of frailty and cognitive impairment separately versus jointly with 5-year cumulative incidence ADL disability. Frailty was defined by the Fried's Phenotype. Cognitive impairment (CI) was defined based the Clock Drawing Test and Immediate and Delayed Word Recall. We used the Fine \& Gray competing risk regression model to account for competing mortality. Frailty alone was a stronger predictor of ADL disability $(\mathrm{HR}=2.31, \mathrm{P}<0.01)$ than cognitive impairment alone $(\mathrm{HR}=1.68, \mathrm{p}<0.01)$; and having both was associated with highest risk $(\mathrm{HR}=3.13, \mathrm{p}<0.01)$. While having $\mathrm{CI}$ alone was associated with ADL-disability-free mortality $(\mathrm{HR}=1.55, \mathrm{p}<0.01)$, having frailty, with or without $\mathrm{CI}$, was not $(\mathrm{HR}=1.34,1.15, \mathrm{p}=0.28,0.66)$, suggesting that frail older adults are at high risk of losing ADL-independence before death. Therefore, preventing and treating frailty holds great promise for achieving the goal of compression of morbidity.

\section{HIERARCHICAL DEVELOPMENT OF PHYSICAL FRAILTY AND COGNITIVE IMPAIRMENT: CLUES INTO ETIOLOGICAL PATHWAYS}

N. Chu ${ }^{1}$, J. Tian, $\mathrm{MS}^{2}$, A.L. Gross, $\mathrm{PhD}^{3}$,

K. Bandeen-Roche, $\mathrm{PhD}^{4}$, M.C. Carlson, $\mathrm{PhD}^{5}$, Q. Xue, $\mathrm{PhD}^{6}$, 1. Johns Hopkins Bloomberg School of Public Health, Bethesda, Maryland, United States, 2. Department of Biostatistics, Bloomberg School of Public Health, Johns Hopkins University, Baltimore, MD, USA; Center on Aging and Health, Johns Hopkins Medical Institutions, Baltimore, MD, USA, 3. Department of Epidemiology, Bloomberg School of Public Health, Johns Hopkins University, Baltimore, MD, USA; Center on Aging and Health, Johns Hopkins Medical Institutions, Baltimore, MD, USA, 4. Department of Biostatistics, Bloomberg School of Public Health, Johns Hopkins University, Baltimore, MD, USA; Center on Aging and Health, Johns Hopkins Medical Institutions, Baltimore, MD, USA, 5. Department of Mental Health, Bloomberg School of Public Health, Johns Hopkins University, Baltimore, MD, USA; Center on Aging and Health, Johns Hopkins Medical Institutions, Baltimore, MD, USA, 6. Division of Geriatric Medicine and Gerontology, Department of Medicine, Johns Hopkins University, Baltimore, MD, USA; Center on Aging and Health, Johns Hopkins Medical Institutions, Baltimore, MD, USA
Frailty and cognitive impairment (CI) are associated and often coexist in older adults. Whether temporal patterns of occurrence reflect different etiologies remain unknown. NHATS participants were assessed annually (2011-2016) for frailty (Fried's criteria) and CI (bottom quintile of clock drawing test or immediate and delayed recall; proxy-report of diagnosis of dementia or AD8 score of $>=2$ ). We used the Fine \& Gray competing risk model to identify predictive factors of frailty onset before CI, CI onset before frailty, and frailty and $\mathrm{CI}$ co-occurrence (within the same visit), accounting for death as competing risk. Of 7,439 community-dwelling participants, $3,839(51.6 \%)$ were free of frailty and $\mathrm{CI} /$ dementia at baseline (median follow-up $=5$ years, $\mathrm{SD}=2$ ). Incident dementia was predictive of frailty onset before CI (sub-distribution hazard ratio $(\mathrm{SHR})=0.37, \mathrm{p}<0.001)$, CI onset before frailty (SHR=2.64, $\mathrm{p}<0.001)$, and frailty and CI co-occurrence (SHR=8.72), $\mathrm{p}<0.001)$. Number of comorbidities was only strongly associated with frailty occurrence first $(\mathrm{p}<0.05)$.

\section{ROLE OF MULTISYSTEM PHYSIOLOGICAL DYSREGULATION IN COGNITIVE IMPAIRMENT AND FRAILTY}

A. Gross ${ }^{1}$, N. Chu, $\mathrm{MPH}^{2}$, M.C. Carlson, $\mathrm{PhD}^{3}$, Q. Xue, $\mathrm{PhD}^{3}$, 1. Johns Hopkins Bloomberg School of Public Health, Baltimore, Maryland, United States, 2. Department of Epidemiology, Johns Hopkins Bloomberg School of Public Health, Baltimore, Maryland, USA, 3. Department of Mental Health, Johns Hopkins Bloomberg School of Public Health, Baltimore, Maryland, USA; Center on Aging and Health, Johns Hopkins Medical Institutions, Baltimore, MD, USA, 3. Division of Geriatric Medicine and Gerontology, Department of Medicine, Johns Hopkins University, Baltimore, MD, USA; Center on Aging and Health, Johns Hopkins Medical Institutions, Baltimore, MD, USA

Although inflammation underlies both dementia and frailty, the role of dysregulation across multiple physiological systems in the genesis of these two syndromes is unclear. We used data from the Women's Health and Aging Study II $(\mathrm{N}=230)$ to derive, via factor analysis, a multisystem physiological dysregulation factor from 12 serum biomarkers of stress response and energy metabolism. We used multinomial logistic regression to predict onset of (1) dementia only, (2) frailty only, and (3) both over 9 years. Compared to women who never developed dementia or frailty, greater physiological dysregulation was associated with incidence of dementia $(\mathrm{OR}=1.42)$, frailty $(\mathrm{OR}=1.77)$, and both $(\mathrm{OR}=2.34)$. After adjustment for IL-6, physiological dysregulation was no longer associated with incidence of dementia $(\mathrm{OR}=1.01)$ but remained associated with onset of frailty $(\mathrm{OR}=1.46)$ and frailty/dementia $(\mathrm{OR}=1.74)$. These data suggest inflammation may be a common risk factor for dementia and frailty, but multisystem physiological dysregulation appears specific to frailty onset.

\section{JOINT TRAJECTORIES OF COGNITION AND} FRAILTY, AND ASSOCIATED BURDEN OF PATIENTREPORTED OUTCOMES

Z. $\mathrm{Liu}^{1}$, L. Han, $\mathrm{PhD}^{2}$, E.A. Gahbauer, MD, $\mathrm{MPH}^{3}$, H.G. Allore, $\mathrm{PhD}^{4}$, T.M. Gill, $\mathrm{MD}^{5}$, 1. Yale University, New Haven, Connecticut, United States, 2. Department of Internal Medicine, Yale School of Medicine, New Haven, 
CT, USA, 3. Department of Internal Medicine, Yale School of Medicine, New Haven, CT, USA, 4. Department of Internal Medicine, Yale School of Medicine, New Haven, CT, USA, 5. Department of Internal Medicine, Yale School of Medicine, New Haven, CT, USA

Longitudinal data (Year 1998-2009) from 690 community-living persons 70 years or older were used to evaluate joint trajectories of cognition (MMSE score) and frailty (Fried's phenotype) and their association with the cumulative burden of patient-reported outcomes (i.e. hospitalization, nursing home admission, and disability). Four joint trajectories were identified, including "No cognitive frailty" $(27.8 \%)$, "Slow cognitive decline and progressive frailty" $(45.5 \%)$, "Accelerated cognitive decline and progressive frailty" $(20.2 \%)$, and "Early cognitive decline and progressive frailty" (termed "Cognitive Frailty", 6.5\%). The "No cognitive frailty" group had the lowest cumulative burden of all patient-reported outcomes, whereas the "Cognitive frailty" group had the highest cumulative burden with the exception of hospitalization. We concluded that community-living older persons exhibit distinct joint trajectories of cognition and frailty and experience an increasing burden of nursing home admission and disability as they age, with the greatest burden for those on a "Cognitive frailty" trajectory.

\section{SESSION 565 (SYMPOSIUM)}

\section{ADDRESSING ECONOMIC INSECURITY IN LATER LIFE: RESPONSES AND OPTIONS}

Chair: J.E. Mutchler, University of Massachusetts Boston, Boston, Massachusetts

As Americans live longer, multi-faceted and creative strategies for promoting economic security in later life are required. Economic security signals a balance between income and expenditures; accordingly, promoting economic security in later life invites innovation and adjustments in income, in expenses, or both. In this symposium we discuss strategies for combatting economic insecurity, focusing on housing, healthcare, employment, savings, financial literacy and planning. Because housing and healthcare represent the largest share of financial burden facing older adults, addressing housing and healthcare costs will have the biggest impact on the expenditure side of the economic security equation. Economic security can also be promoted through working longer, including building new careers through self-employment or entrepreneurship. Other strategies such as encouraging comprehensive savings and efforts that enhance financial literacy and planning can help older adults accumulate wealth and make wiser financial decisions in order to realize greater benefit from existing assets. As well as sharing their research on these issues, participants in this panel offer examples of promising policy interventions that can help support economic security in later life.

\section{ADDRESSING ECONOMIC INSECURITY IN LATER LIFE: RESPONSES AND OPTIONS}

T. Neuman, Kaiser Family Foundation, Washington, District of Columbia, United States

Health care costs can have a major impact on economic security in retirement. Medicare provides coverage to virtually all Americans age 65 and older, but gaps in coverage put older adults at risk. Medicare does not cover the high costs of extensive care in a nursing homes, assisted living facilities, or at home. Traditional Medicare includes neither a cap on out-of-pocket expenses, nor coverage for dental, vision, or hearing. Medicare Part D has helped reduce the cost of medications, but even so, many older Americans incur significant out-of-pocket costs for their prescriptions drugs. Our analysis, based on several studies, finds older adults paying more than younger adults for health as a share of household budgets, with health care costs consuming a significant and rising share of Social Security and total income. This presentation will synthesize research that documents these concerns, and discuss related issues and policy options.

\section{PROPERTY TAX DEFERRAL: A PROPOSAL TO HELP MASSACHUSETTS SENIORS}

A.N. Walters ${ }^{1}$, A.H. Munnell, $\mathrm{PhD}^{2}$, A. Belbase, $\mathrm{MPA}^{3}$, W. Hou, $\mathrm{MS}^{4}, 1$. Center for Retirement Research at Boston College, Chestnut Hill, Massachusetts, United States, 2. Center for Retirement Research at Boston College, Chestnut Hill, MA, USA, 3. Center for Retirement Research at Boston College, Chestnut Hill, MA, USA, 4. Center for Retirement Research at Boston College, Chestnut Hill, MA, USA

Many retirees in Massachusetts do not have enough income to meet their basic living expenses. Social Security will provide less to pre-retirement earnings and personal retirement savings are meager. Increasingly, retirees will need to tap their home equity to make ends meet. Many could use their home, but do not want to downsize or take out a reverse mortgage. An alternative way to tap home equity is by deferring property taxes - one year at a time, no up-front costs, and easy to understand. Massachusetts has a program but it is administered locally and most seniors are either ineligible or do not know about it. This proposal outlines a statewide program with broader eligibility, outreach, and simplified enrollment.

\section{THE ROLE OF GRADUAL RETIREMENT IN THE ECONOMIC SECURITY OF OLDER AMERICANS}

K.E. Cahill, Center on Aging \& Work at Boston College, Boise, Idaho, United States

This research explores the role gradual retirement plays in the retirement income security of older Americans. Gradual retirement encompasses three pathways to labor force withdrawal: phased retirement, a reduction in hours with one's current employer; bridge employment, a switch to a new employer prior to labor force exit; and reentry, a return to work following an initial retirement. Each of these pathways plays a unique role in promoting economic security later in life. For example, in light of the many uncertainties that come with retirement, reentry can provide a critical backstop for financial security through the optionality of continued work. Using longitudinal data on four cohorts of older Americans from the Health and Retirement Study $(n=10,779)$ this research explores each of the retirement transition types and how they impact retirement income security, and then presents policy options to help facilitate gradual retirement for interested workers. 